\title{
Preoperative octreotide therapy in acromegaly: Associations between effects on glucose homeostasis and biochemical cure
}

\author{
Helseth $R^{1}$, Carlsen $S M^{2,3}$, Bollerslev $\mathrm{J}^{4,5}$, Svartberg $\mathrm{J}^{6,7}, \varnothing \mathrm{ksnes} \mathrm{M}^{8}$, Skeie $\mathrm{S}^{9}$ and Fougner $\mathrm{SL}^{2}$
}

${ }^{1}$ Department of Internal Medicine, Drammen Hospital, Vestre Viken, NORWAY, ${ }^{2}$ Department of Endocrinology, Medical Clinic, St. Olavs University Hospital, Trondheim, ${ }^{3}$ Unit for Applied Clinical Research, Norwegian University of Science and Technology (NTNU), Trondheim, "4 Section of Specialized Endocrinology, Department of Endocrinology, Oslo University Hospital, Rikshospitalet, Oslo, ${ }^{5}$ Faculty of Medicine, University of Oslo, Oslo, ${ }^{6}$ Division of Internal Medicine, University Hospital of North Norway, Tromsø, ${ }^{7}$ Tromsø Endocrine Research Group, Institute of Clinical Medicine, UiT The Arctic University of Norway, Tromsø, ${ }^{8}$ Department of Medicine and Centre for Clinical Research, Haukeland University Hospital, Bergen, 9 Department of Endocrinology, Stavanger University Hospital, Stavanger, NORWAY

\section{BACKGROUND AND OBJECTIVE}

In acromegaly, high GH/IGF-1 levels are associated with abnormal glucose metabolism. Treatment with somatostatin analogues (SSAS) reduces the GH and IGF-1 levels. However, SSAs may worsen glucose homeostasis despite this, due to concomitant inhibition of insulin secretion.

The objective of this study was to analyse the possible association between glucose homeostasis, SSA treatment and biochemical cure in de novo patients with acromegaly.

\section{DESIGN}

Post hoc analysis from a randomised controlled trial of newly diagnosed patients with acromegaly in Norway during $1999-2004$ (the POTA study, ref 1).

\section{METHODS}

Of the 62 de novo patients included in the POTA study, seven subjects (11\%) had impaired glucose tolerance and 12 subjects (19 $\%$ ) had overt diabetes mellitus.

The 55 patients with acromegaly not using antidiabetic medication were included in the present analyses. Of them, 26 received SSA for 6 months preoperatively. HbA1c and an oral glucose tolerance test (OGTT) were performed at diagnosis, before surgery and 3 months postoperatively. Area under curve of glucose (AUCglucose) was calculated. Indices of glucose homeostasis were compared between cured and non-cured patients.

\section{RESULTS}

No associations between basal IGF-1/GH levels and fasting glucos AUCglucose or $\mathrm{HbA1C}$ were found.

After SSA treatment, the percentage reduction in both mean $\mathrm{GH}$ IGF-1 correlated positively with reduction in HbAlc levels (Table.

\begin{tabular}{lccccc} 
& \multicolumn{2}{c}{ Mean GH reduction } & \multicolumn{2}{c}{ IGF-1 reduction } \\
& $\mathrm{r}$ & $\mathrm{p}$ & $\mathrm{r}$ & $\mathrm{p}$ \\
& & & & \\
Fasting glucose (\% reduction) & 0.00 & 0.99 & 0.03 & 0.91 \\
HbA1c (\% reduction) & 0.50 & $0.021^{\star}$ & 0.64 & $0.001^{\star}$ \\
AUCglucose (\% reduction) & 0.13 & 0.56 & 0.050 & 0.82
\end{tabular}

Table 1. After 6 months SSA pretreatment. Correlations between changes in hormone levels and changes in glucose homeostasis.

\section{RESULTS cont.}

Biochemical cure by IGF-1 and nadir GH, or IGF-1/nadir GH alone after SSA treatment were also associated with larger reduction in $\mathrm{HbAlC}$ but not to AUCglucose or fasting glucose (Table 2, figure 1).

\begin{tabular}{lcccccc}
\hline & \multicolumn{4}{c}{$\begin{array}{c}\text { Cured by both nadir } \\
\text { GH and IGF-1 }\end{array}$} & $\begin{array}{c}\text { Cured by } \\
\text { nadir GH }\end{array}$ & $\begin{array}{c}\text { Cured by } \\
\text { IGF-1 }\end{array}$ \\
\hline & Yes & No & $p$ & $p$ & $p$ \\
\hline Fasting glucose (\% reduct.) & 0.0 & -3.9 & 0.18 & 0.93 & 0.47 \\
HbA1c (\% reduction) & 5.3 & -5.1 & $0.003^{\star}$ & $0.009^{\star}$ & $0.03^{\star}$ \\
AUCglucose (\% reduction) & -13.6 & -12.3 & 0.89 & 0.49 & 0.55 \\
\hline
\end{tabular}

Table 2. Percentage reduction in glucose indices (median value) and correlation to biochemical cure after 6 months octreotide treatment. Mann-Whitney test.

Table 3 (below). Correlation to biochemical cure 3 months after surgery.

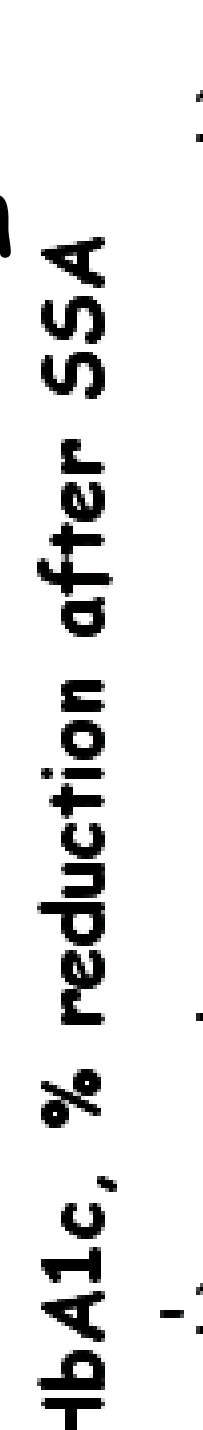

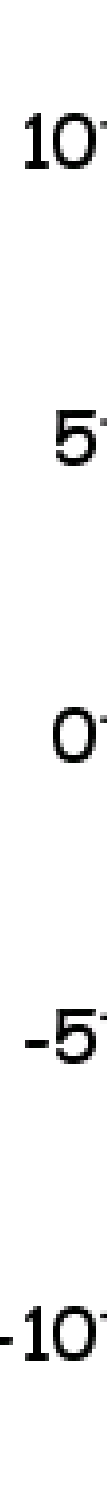

$-15-$

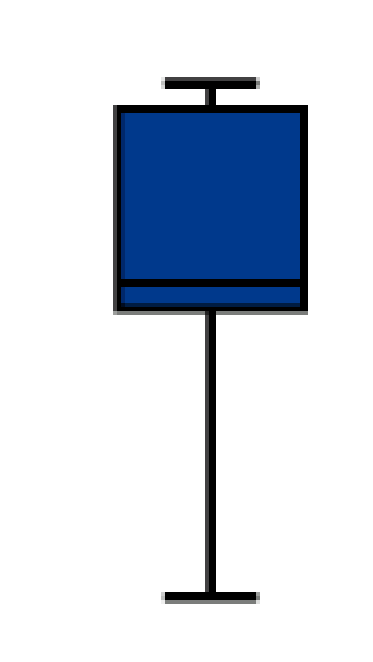

Fasting glucose (\% reduct.) HbA1c (\% reduction) AUCglucose (\% reduction) Aucglucose (\% reduction)

Figure 1. After SSA pretreatment Percentage reduction in $\mathrm{Hb} A 1 \mathrm{c}$ in patients with and without achieved biochemical cure.
No

Biochemical cure after SSA I nree montns postoperatively, biochemical cure by IGF-1 and nadir $G H$ associated with lower AUC-glucose $(p=0.041)$ but not with fasting glucose or the reduction from baseline in both variables. For $\mathrm{HbA1c}$, a possible trend towards larger reduction from baseline in patients cured after surgery, can be claimed (Table 3).

\section{CONCLUSION}

In de novo patients with acromegaly, disease activity do not correspond to glucose homeostasis indices.

However, hormonal control of GH and IGF-1 after SSA

treatment is associated with reduction in $\mathrm{HbAlc}$. 